\title{
LA INNOVACIÓN Y LA MIGRACIÓN CALIFICADA EN LA ENCRUCIJADA: REFLEXIONES A PARTIR DE LA EXPERIENCIA MEXICANA
}

\author{
Innovation and highly skilled migration at a critical junction: \\ reflections based on the Mexican experience
}

\author{
Raúl Delgado Wise \\ Mónica Guadalupe Chávez Elorza² \\ Héctor Rodríguez Ramírez ${ }^{3}$
}

\begin{abstract}
Resumen. Se analiza la migración calificada desde un prisma analítico que hasta ahora había sido esencialmente ignorado en la literatura especializada: la profunda reestructuración que experimentan los sistemas de innovación en el marco de la globalización neoliberal y bajo la batuta de Estados Unidos. Además de dilucidar las principales transformaciones que acusan los sistemas de innovación a nivel mundial, se examina el incremento explosivo que han tenido las patentes en el curso de las últimas dos décadas, el cual ha venido acompañado de una creciente participación de científicos y tecnólogos proveniente de países periféricos y emergentes. A partir de esta mirada analítica y tomando como referente el caso de la migración calificada mexicana, se aportan algunos elementos tendientes a desentrañar las nuevas modalidades de intercambio desigual que emergen en el horizonte Norte-Sur y que tienen como trasfondo una mercantilización y apropiación privada sin precedentes del conocimiento, en tanto bien común intangible.
\end{abstract}

Palabras clave: migración calificada, innovación, patentes, globalización neoliberal, bienes comunes intangibles.

Abstract. Skilled migration is analyzed through an analytical framework that so far has been essentially ignored in the specialized literature on the subject: the deep restructuration process to which

1 Profesor Investigador, Coordinador de la Cátedra UNESCO de Migración, Desarrollo y Derechos Humanos. Zacatecas, ZC, México.

2 Investigadora Postdoctoral Conacyt, Unidad Académica en Estudios del Desarrollo, UAZ. Zacatecas, ZC, México.

3 Profesor Investigador, Escuela de Gobierno y Transformación Pública, Tecnológico de Monterrey, Campus Monterrey. Monterrey, NL, México. 
innovation systems have been subjected under the context of neoliberal globalization and the leadership of the United States. Besides unraveling the main transformations undertaken by innovation systems worldwide, the explosive growth of patents that has taken place during the last two decades is analyzed. This trend has been accompanied by an increasing participation of scientists and technicians from peripheral and emerging countries. From this particular analytical lens and taking as a reference the Mexican case, several elements aimed at disentangling the new modalities of unequal exchange that have emerged along the North-South divide are provided. At the background of this embryonic trend underlies an unprecedented commodification and appropriation of knowledge, as an intangible common good.

Keywords: skilled migration, innovation, patents, neoliberal globalization, intangible common goods.

\section{Introducción}

A partir de la década de 1990 la migración altamente calificada experimenta ritmos de crecimiento que rebasan, con mucho, los correspondientes a la migración internacional. Ello cobra particular relevancia en el caso de los países periféricos o emergentes y se relaciona de manera significativa con las transformaciones que experimentan los sistemas de innovación a nivel global. Profundizar en torno a esta problemática, la cual ha recibido relativamente poca atención en la literatura, constituye el objetivo central de este trabajo. Nos interesa, ante todo, examinar: a) las transformaciones que en décadas recientes han experimentado los sistemas de innovación, tomando como referente el caso paradigmático de Silicon Valley; b) dimensionar el significado y alcances de estos cambios a partir del curso que han seguido las patentes, destacando la creciente participación de científicos y tecnólogos provenientes de países periféricos, y, a partir de este prisma analítico, c) discernir las causas y características de la creciente selectividad que acusan las migraciones internacionales, particularmente en dirección sur-norte, tomando como referente el caso de México.

Nuestra intención es trascender las visiones dominantes acerca del nexo entre migración calificada y desarrollo, las cuales varían desde la llamada fuga de cerebros, hasta las nociones en boga de circulación y ganancia de cerebros. Se trata, en esencia, de visiones descontextualizadas, que no logran dilucidar la naturaleza de las relaciones de intercambio desigual que caracterizan a la globalización neoliberal y, por consiguiente, que pierden de vista las crecientes contradicciones entre el desarrollo científico-tecnológico, la satisfacción de las necesidades sociales y la preservación del medio ambiente que se generan en el horizonte norte-sur. Bajo esta vertiente analítica, la recuperación crítica de la noción de bienes comunes tangibles e intangibles está abriendo una rica veta analítica en el debate latinoamericano, la cual es referida sucintamente en el 
apartado final del trabajo, con miras a perfilar rutas alternas de investigación en la materia.

\section{Reestructuración de los sistemas de innovación}

El contexto en el que se despliega la migración calificada bajo el andamiaje de la globalización neoliberal ${ }^{4}$, particularmente aquella proveniente de los países periféricos o emergentes, está signado por una profunda reestructuración de los sistemas de innovación a escala global, con Estados Unidos a la cabeza y teniendo a las grandes corporaciones multinacionales como agentes centrales. Cuatro dimensiones estratégicas caracterizan esta reestructuración:

1. Una mayor internacionalización y fragmentación de las actividades de investigación y desarrollo. En contraposición a los esquemas tradicionales de invención "a puertas cerradas" en departamentos de investigación y desarrollo enclavados al seno de las grandes corporaciones, a esta tendencia se le conoce como innovación abierta en referencia a la apertura y redistribución espacial de funciones corporativas intensivas en conocimiento con la creciente participación de socios externos, tales como proveedores, clientes, subcontratistas, universidades y centros de investigación, para dar paso a la configuración y reconfiguración de ecosistemas o redes de innovación ${ }^{5}$. Bajo esta nueva vertiente se asume "...que el conocimiento útil es ampliamente distribuido, y que aún las instituciones de I y D más competentes deben identificar, conectarse a y potenciar fuentes de conocimiento externo como procesos nodales de la innovación" ${ }^{\prime \prime}$.

2. El establecimiento de ciudades científicas -como Silicon Valley en Estados Unidos y los nuevos "Silicon Valley" instaurados en regiones periféricas o emergentes, principalmente en Asia- donde se crean sinergias colectivas para acelerar las dinámicas de innovación ${ }^{7}$. Se trata, en el fondo, como lo destaca Annalee Saxenian ${ }^{8}$ de un nuevo paradigma, que se aparta de los viejos modelos "cerrados" de investigación y desarrollo incrustados en las grandes

4 Entre los rasgos más conspicuos de la globalización neoliberal, sobresalen: el predominio del capital monopolista financiero, la configuración de redes globales de capital monopolista (como estrategia de internacionalización de la producción, los servicios y el comercio por las grandes corporaciones multinacionales), el extractivismo y el acaparamiento y control de la tierra directa o indirectamente por los agronegocios. Ello ha dado lugar a nuevas formas rentistas y espurias de ganancia extraordinaria y propiciado una embestida global en contra de la clase trabajadora y sectores populares, lo que a su vez ha desencadenado un incremento de las desigualdades sociales y una profundización, sin precedentes, del desarrollo desigual a escala planetaria, más detalles en DELGADO-WISE, Raúl, MARTIN, David. La economía política del arbitraje laboral global: capital y trabajo en el contexto de la globalización neoliberal.

OECD. Open Innovation in Global Networks.

6 CHESBROUGH, Henry. Open Innovation: A New Paradigm for Understanding Industrial Innovation.

7 STURGEON, Timothy J. What really goes on in Silicon Valley? Spatial clustering and dispersal in modular production networks.

8 SAXENIAN, Annelee. Regional Advantage: Culture and Competition in Silicon Valley and Route 128; IDEM. Local and Global Networks of Immigrant Professsionals in Silicon Valley. 
corporaciones y que abre el camino hacia una nueva cultura de la innovación basada en la flexibilidad, descentralización e incorporación, bajo diferentes modalidades, de nuevos y cada vez más numerosos jugadores que interactúan en espacios locales y transnacionales. En este último ámbito, las plataformas de innovación que se establecen en los países periféricos tienden a operar como extensiones de las plataformas instauradas en los países centrales, aprovechando ventajas salariales, tributarias y de otro tipo, lo que les confiere el carácter de lo que concebimos como maquiladoras científicas ${ }^{9}$.

3. El desarrollo de nuevas formas de control de las agendas de investigación mediante capital de riesgo, asociaciones y subcontrataciones, entre otros y de apropiación de los productos del trabajo científico vía adquisición de patentes por las grandes corporaciones multinacionales, a través del Ilamado strategic investment. Resulta interesante observar al respecto que, aun cuando $76 \%$ de las patentes a universidades y alrededor de la mitad del total de patentes en años recientes en Estados Unidos fueron atribuidas a un inventor foráneo, 93\% de los registros de las mismas fueron otorgados, como veremos en el siguiente apartado, a corporaciones multinacionales ${ }^{10}$.

4. La expansión en el horizonte Sur-Norte de la fuerza de trabajo altamente calificada, en particular en las áreas de ciencia e ingeniería y el creciente el reclutamiento -vía partnerships, outsourcing y offshoring- de esa fuerza de trabajo con especial significación en el caso de los países periféricos ${ }^{11}$. De hecho, la evolución de esta dinámica de reestructuración ha cristalizado en una nueva geografía de la innovación, así como de la investigación y el desarrollo científico/tecnológico asociados a ella. Desde 2011, Estados Unidos fue superado por China, Japón, Corea del Sur e India como principal inversor en investigación y desarrollo, y se espera que en 10 años más China supere a Estados Unidos en este renglón ${ }^{12}$. En lo referente a fuerza científico/tecnológica (brain power), de acuerdo con la American Community Survey en 2011, 1 de cada 3 Masters y 1 de cada 2 doctores en ciencias e ingeniería en Estados Unidos era extranjero -principalmente de países periféricos o emergentes-, al tiempo que fuera de Estados Unidos, destacadamente en China y la Unión Europea, se estaban preparando mayores contingentes de doctores en estas áreas del conocimiento que en el coloso de norte América ${ }^{13}$. Con todo, la propiedad intelectual sigue estando concentrada abrumadoramente, como veremos enseguida, en manos de Estados Unidos.

9 GALLENGHER, Kevin, ZARSKY, Lyuba. The Enclave Economy: Foreign Investment and Sustainable Development in Mexico's Silicon Valley.

10 NATIONAL SCIENCE BOARD Science and Engineering Indicators Digest; RAND CORPORATION. U.S. Competitiveness in Science and Technology; USPTO. Patenting by Organizations.

11 BATTELLE. 2013 Global R\&D Funding Forecast; MORIS, Franciso, KANNANKUTTY, Nirmala. New employment statistics from 2008 Business R\&D and Innovation Survey.

12 BATTELLE, op. cit.

${ }^{13}$ FREEMAN, Richard. The new global labor market; GALAMA, Titus, HOSEK, James. U.S. Competitiveness in Science and Technology. 
Es pertinente subrayar que esta dinámica de reestructuración ha posibilitado a las grandes corporaciones multinacionales poner a un creciente contingente de trabajadores científico-tecnológicos del sur a su servicio, transferir riesgos y responsabilidades y capitalizar ostensibles beneficios mediante la concentración de patentes. Bajo esta nueva trama se ha venido produciendo una mercantilización y monopolización sin precedentes del trabajo científico, con una visión de corto plazo y ajena a toda preocupación social, donde la emigración física e indirecta de personal altamente calificado de los países periféricos o emergentes desempeña un papel cada vez más significativo.

\section{La creciente mercantilización y privatización del conocimiento a través de las patentes}

En el curso de las últimas dos décadas la dinámica de acumulación de capital se sintetiza en un nuevo lema, por lo demás engañoso y ajeno a una visión positiva y equitativa del progreso: patentad, patentad. En efecto, como se aprecia en la figura 1, el número de patentes registradas en Estados Unidos se incrementó ostensiblemente: poco más de 4 millones a partir de 1991, lo que supera el número de patentes acumulado a lo largo de los tres siglos previos.

\section{Figura 1 - Evolución de las patentes registradas en Estados Unidos}

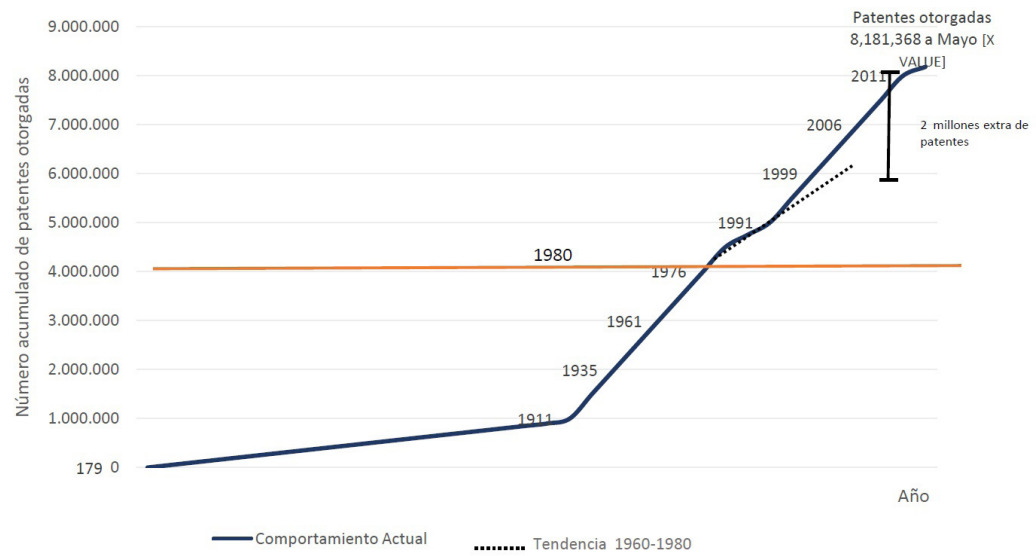

Fuente: SMITH, Gina. Can the U.S. patent system be saved? ComputerWold, 2012. Disponible en < http://www.computerworld.com/article/2505817/it-management/can-the-u-s--patent-system-besaved-.html>. Consultado el 10.10.2015.

Esta impresionante tendencia que se observa en Estados Unidos, se corrobora a través de la información disponible sobre solicitudes de patentes a nivel mundial aportada por la Patent Cooperation Treaty (PCT) de la World 
International Patent Organization (WIPO) ${ }^{14}$, donde, como se desprende del cuadro 1, entre 1991 y 2010 se concentraron el 92\% de las solicitudes realizadas desde 1981. Cabe subrayar que en 2010 la PTC cubrió el 54\% del total de patentes que se solicitaron a nivel global ${ }^{15}$.

\section{Cuadro 1 - PCT-WIPO* 1981-2010: Total de solicitudes de patentes a nivel mundial y tasa emigratoria}

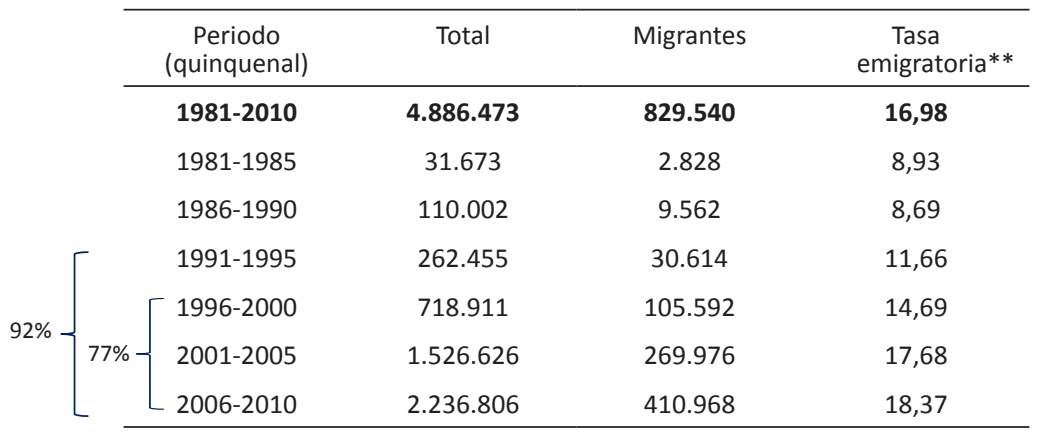

Nota: *Patent Cooperation Treaty (PCT), World International Patent Organization (WIPO). **Tasa emigratoria de solicitudes de patentes es igual al cociente del número de solicitudes hechas por emigrantes dividido por la suma de solicitudes hechas por nacionales y emigrantes.

Fuente: Estimaciones propias con datos de MIGUÉLEZ, Ernest, FINK, Carsten. Measuring the international mobility of inventors: a new database.

Más todavía, de acuerdo con la misma fuente (PTC), Estados Unidos despunta como la primera potencia capitalista del mundo en materia de innovación, al concentrar $28 \%$ de la totalidad de patentes gestionadas. Al considerar al total de países de la OECD -sin incluir a México, Chile y Turquíase aprecia que conjuntamente concentran el $90 \%$ de las solicitudes mundiales de patentes, lo que revela que la propiedad intelectual continúa figurando como un rasgo distintivo del llamado mundo desarrollado. $Y$ resulta igualmente destacable, aunque en sentido opuesto, el extremadamente bajo nivel de participación de América Latina y el Caribe en la generación de patentes, cuyo aporte al cúmulo global de solicitudes consignadas fue de apenas el $0.7 \%$ (véase cuadro 2).

${ }^{14}$ El Tratado de Cooperación en materia de Patentes (Patent Cooperation Treaty) es un tratado internacional administrado por la Organización Mundial de la Propiedad Intelectual (World Intellectual Property Organization, WIPO) que ofrece a los solicitantes de patentamiento un camino ventajoso para buscar la protección internacional de patentes. El tratado entró en vigor en 1978 con solo 8 miembros, para 2012 había 146 estados miembros y actualmente hay 148 países miembros; México se adhirió al tratado en 1995.

${ }^{15}$ MIGUÉLEZ, Ernest, FINK, Carsten. Measuring the international mobility of inventors: a new database. 


\section{Cuadro 2 - PCT-WIPO* 1996-2010: Países con mayor número de solicitudes de patentes en el mundo y en Latinoamérica}

\begin{tabular}{|c|c|c|c|c|c|}
\hline País & $\begin{array}{c}\text { Total } \\
\text { solicitudes } \\
\text { patentes }\end{array}$ & $\begin{array}{c}\text { Tasa } \\
\text { emigratoria }\end{array}$ & País & $\begin{array}{c}\text { Total } \\
\text { solicitud } \\
\text { patentes }\end{array}$ & $\begin{array}{c}\text { Tasa } \\
\text { emigratoria }\end{array}$ \\
\hline Total Global & $4,482,343$ & 9.6 & \multirow{2}{*}{$\begin{array}{c}\text { Total Latinoamérica } \\
\text { y El Caribe }\end{array}$} & \multirow{2}{*}{30,366} & \multirow{2}{*}{33.7} \\
\hline Total OCDE** & $4,032,186$ & 6.3 & & & \\
\hline $\begin{array}{l}\text { Primeros diez } \\
\text { países }\end{array}$ & $3,673,953$ & 6.7 & $\begin{array}{c}\text { Primeros seis países } \\
\text { de Latinoamérica } \\
\text { y El Caribe }\end{array}$ & 26,778 & 28.2 \\
\hline Estados Unidos & $1,237,060$ & 1.3 & Brasil & 12,779 & 16.9 \\
\hline Japón & 710,516 & 1.1 & México & 6,335 & 32.6 \\
\hline Alemania & 627,460 & 6.3 & Argentina & 2,966 & 54.9 \\
\hline Reino Unido & 216,480 & 17.4 & Colombia & 1,673 & 56.3 \\
\hline Francia & 212,571 & 10.9 & Chile & 1,522 & 36.1 \\
\hline China & 208,665 & 29.7 & Cuba & 1,503 & 15.4 \\
\hline República de Corea & 183,584 & 5.4 & & & \\
\hline Canadá & 102,917 & 25.5 & & & \\
\hline Países Bajos & 93,105 & 12.9 & & & \\
\hline Suecia & 81,595 & 6.4 & & & \\
\hline \multicolumn{3}{|c|}{ Periodo 1996-2010: OCDE 90.0\% del Global } & \multicolumn{2}{|c|}{$\begin{array}{l}\text { AL y Caribe } 0.68 \% \text { del Clobal } \\
\text { AL y Caribe } 0.75 \% \text { de la OCDE }\end{array}$} & \\
\hline
\end{tabular}

Nota: *Patent Cooperation Treaty (PCT), World International Patent Organization (WIPO).

**No incluye a México, Chile ni a Turquía.

Fuente: Estimaciones propias con datos de MIGUÉLEZ, FINK, op. cit.

Otro aspecto del cuadro 2 que llama la atención es el hecho de que la tasa de solicitudes de patentamiento de los latinoamericanos y caribeños que residen en el extranjero (principalmente en países desarrollados) supera 4.5 veces la tasa correspondiente a los países de la OECD y 3 veces la media mundial. Y en este contexto, aunque Brasil y México concentran el $71 \%$ de las patentes de la región, sus comportamientos en materia de innovación resultan contrastantes: mientras que Brasil duplica prácticamente a México por el número de patentes, lo inverso ocurre al considerar las tasas de migración de uno y otro país.

Aunado a las cifras referidas, en sí mismas reveladoras del creciente y desbordante ritmo de patentamiento y de las desigualdades en su distribución por países y regiones, resulta importante consignar que existe una notable diferencia entre los inventores (según su país de origen) y los propietarios de las patentes (individuos, sector público o corporaciones). Al respecto, según datos del Patent Cooperation Treatment, el grueso de solicitudes realizadas en los últimos 15 años correspondió a corporaciones, particularmente a grandes corporaciones multinacionales, las cuales acumularon poco más de tres cuartas 
partes de patentes solicitadas (véase figura 2). No resulta sorprendente, por tanto, que dicha tendencia coincida con la que exhiben los países de la OECD, quienes, como vimos, son los que concentran el mayor número de patentes a nivel planetario.

\section{Figura 2 - PCT-WIPO* 1996-2010: Solicitudes de patentes según tipo de solicitante}

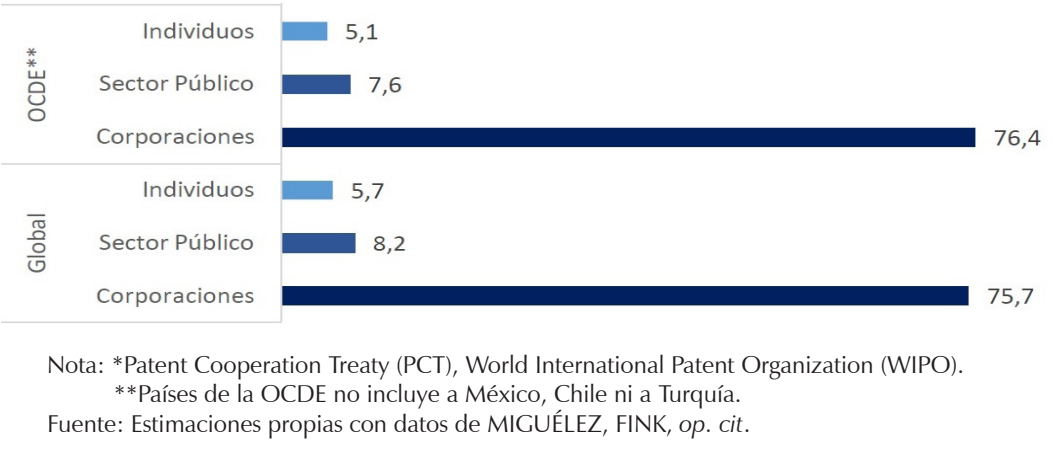

Cabe subrayar que en el caso de Estados Unidos, epicentro de la innovación global, las corporaciones -sean nacionales o extranjeras- concentraron el 93\% de las patentes otorgadas en 2014 , de las cuales $46 \%$ correspondieron a empresas estadounidenses, $18 \%$ a japonesas, $6 \%$ a alemanas, $5 \%$ a coreanas, $4 \%$ a taiwanesas y $2 \%$ a chinas. Más aún, de las 12 corporaciones más importantes, seis eran estadounidenses (19,294 patentes), cinco japonesas (12,197 patentes) y una coreana $(4,936 \text { patentes })^{16}$.

Por otro lado y en abierto contraste con los elevados niveles de concentración y apropiación de la propiedad intelectual por las grandes corporaciones multinacionales, es importante observar que la participación de inventores de origen extranjero en Estados Unidos se elevó de 18\% en 1963 a $51 \%$ en 2014. Ello significa que, paradójicamente al incremento del poder corporativo derivado de la propiedad intelectual, se ha venido gestando una creciente dependencia en materia de innovación, de la capacidad e inventiva del talento extranjero.

Si se considera, además, que a nivel mundial el segmento de mayor crecimiento entre los inventores extranjeros, es el de los innovadores provenientes de países periféricos o emergentes, queda claro que se está configurando una contratendencia al sentido tradicional de la dependencia que históricamente ha distinguido al mundo desarrollado. No debe perderse de vista, en este sentido, que la tasa de crecimiento anual de las solicitudes de patentes en países de la OECD por inmigrantes provenientes del Sur entre 1990 y 2010 fue de 22.8\%,

${ }^{16}$ USPTO, op. cit. 
superando la tasa anual de crecimiento de inventores provenientes de países de la OECD, que en el mismo periodo fue de $16.4 \%$. A ello habría que agregar el hecho de que en 2010 el porcentaje de solicitantes de patentes provenientes de países periféricos o emergentes fue de $45.8 \%$ (véase cuadro 3 ).

\section{Cuadro 3 - PCT-WIPO*: Flujos de solicitudes de patentes hechas por migrantes, 1990, 2000 y 2010}

\begin{tabular}{|c|c|c|c|c|c|c|c|}
\hline \multirow[t]{2}{*}{ Dirección de flujo } & \multirow{2}{*}{$\begin{array}{c}\text { Periodo } \\
1990 \text { - } \\
2010\end{array}$} & \multicolumn{2}{|c|}{ Año } & \multirow{2}{*}{$\begin{array}{c}\begin{array}{c}\text { Tasa de } \\
\text { crecimiento }\end{array} \\
1990- \\
2010\end{array}$} & \multicolumn{3}{|c|}{ Distribución porcentual } \\
\hline & & 1990 & 2010 & & $\begin{array}{c}1990 \text { - } \\
2010\end{array}$ & 1990 & 2010 \\
\hline Total & 820,072 & 2,922 & 91,720 & 18.8 & 100.0 & 100.0 & 100.0 \\
\hline \multicolumn{8}{|l|}{ Sur - Norte } \\
\hline Países no OCDE a países OCDE & 317,946 & 654 & 39,936 & 22.8 & 38.8 & 22.4 & 43.5 \\
\hline \multicolumn{8}{|l|}{ Norte - Sur } \\
\hline Países OCDE a países no OCDE & 23,598 & 54 & 3,822 & 23.7 & 2.9 & 1.8 & 4.2 \\
\hline \multicolumn{8}{|l|}{ Norte - Norte } \\
\hline Países OCDE a países OCDE & 464,900 & 2,208 & 45,880 & 16.4 & 56.7 & 75.6 & 50.0 \\
\hline \multicolumn{8}{|l|}{ Sur - Sur } \\
\hline Países no OCDE a países no OCDE & 13,628 & 6 & 2,082 & 34.0 & 1.7 & 0.2 & 2.3 \\
\hline
\end{tabular}

Nota: *Patent Cooperation Treaty (PCT), World International Patent Organization (WIPO).

**Países de la OCDE no incluye a México, Chile ni a Turquía.

Fuente: Estimaciones propias con datos de MIGUÉLEZ, FINK, op. cit.

No está por demás agregar que lo hasta aquí consignado pone de relieve los riesgos que subyacen a la carrera desenfrenada en la que están enfrascadas las grandes corporaciones multinacionales en su afán insaciable de apropiación y concentración de patentes. De aquí se derivan dos tipos de desviaciones en la orientación de los procesos de innovación que no pueden ni deben ser soslayados. Por un lado, el hecho de que un número no despreciable de las patentes adquiridas por las corporaciones son administradas por ellas con el propósito de evitar la competencia y conservar su poder monopolista ${ }^{17}$. Por otro lado, la supeditación de los frutos de las innovaciones a la maximización de las ganancias corporativas por encima y a costa de sus potenciales beneficios para la sociedad y en muchas ocasiones también, sin tomar en cuenta los posibles daños que pudiesen ocasionar al medio ambiente. Esta situación ha abierto un interesante y rico campo de debate académico relacionado con la defensa y protección de los bienes comunes tangibles e intangibles ${ }^{18}$.

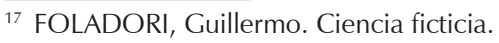

${ }^{18}$ LAVAL, Christian, DARDOT, Pierre. Común. Ensayo sobre la revolución del siglo XXI; VERCELLY, Ariel, THOMAS, Hernán. Repensando los bienes comunes: análisis soco-técnico sobre la construcción y regulación de los bienes comunes; RAMIS, Álvaro. Los bienes comunes intangibles en el capitalismo cognitivo. 


\section{La importancia del fenómeno migratorio y su creciente selectividad: apuntes sobre la experiencia mexicana}

La movilidad humana se ha convertido en un tema prioritario en la agenda internacional. En la actualidad hay 232 millones de migrantes en el mundo, tres cuartas partes de los cuales provienen de países periféricos o emergentes. Si a ello se agregan 740 millones de migrantes internos, alrededor de mil millones de personas viven en un lugar diferente al que nacieron, es decir, uno de cada siete habitantes del planeta es un migrante ${ }^{19}$. Más aún, si se considera que alrededor del $90 \%$ del total de migrantes son laborales ${ }^{20}$, uno de casi tres trabajadores en el mundo se ubican en esta categoría y por lo general están sujetos a condiciones de mayor vulnerabilidad y discriminación en el empleo.

En este contexto y en sintonía con los cambios en los sistemas de innovación y la creciente participación de científicos y tecnólogos de países periféricos y emergentes en la generación de patentes, en las últimas dos décadas se ha generado una creciente selectividad de la migración internacional: mientras que la tasa anual de crecimiento de la migración internacional en general, de 1990 a 2010, fue de $1.8 \%$, la correspondiente a la migración calificada fue de $2.7 \%$ (véase cuadro 4). Al igual que la migración en general, la migración calificada se origina mayoritariamente en países periféricos o emergentes ${ }^{21}$.

\section{Cuadro 4 - Población emigrante con educación terciaria a nivel global, 1990 y 2010}

\begin{tabular}{lccc}
\hline & \multicolumn{2}{c}{ Año } & \multicolumn{1}{c}{$\begin{array}{c}\text { Tasa de } \\
\text { crecimiento }\end{array}$} \\
\cline { 2 - 4 } & \multicolumn{1}{c}{$\mathbf{1 9 9 0}$} & $\mathbf{2 0 1 0}$ & $\mathbf{1 9 9 0 - 2 0 1 0}$ \\
\cline { 2 - 4 } Migrantes totales* $^{*}$ & 154.161 .984 & 220.729 .300 & 1,8 \\
Migrantes con educación terciaria** $^{*}$ & 16.245 .039 & 27.781 .759 & 2,7 \\
Porcentaje de migrantes con educación terciaria & 10,5 & 12,6 & \\
\hline
\end{tabular}

Nota: *Datos provenientes de la UN-DESA, 2013. ** La cifra de 1990 es para la población de 25 años y más y proviene de ARTUC, Erhan et alii. Global Assessment of Human Capital Mobility: The Role of Non-OECD. Destinations. La cifra de 2010 es para la población de 15 años y más proviene de la base DIOC 2010/11.

Fuente: Estimaciones propias con base en los datos UN-DESA. Tables of total migrant stock at mid-year by origin and by major area, region, country or area of destination. 2012 and 2013. Disponible en <http://www.un.org/ en/development/desa/population/migration/data/estimates2/estimatesage.shtml > . Consultado el 20.10.2015;

DIOC 2010/11; ARTUC et alii, op. cit.

${ }_{19}$ IOM. Global Migration Trends: an overview.

${ }^{20}$ ILO. Mainstreaming of Migration in Development Policy and Integrating Migration in the Post2015 UN Development Agenda.

${ }^{21}$ LOZANO-ASCENCIO, Fernando, GANDINI, Luciana. Migración calificada y desarrollo humano en América Latina y el Caribe; DOCQUIER, Frédéroc, RAPOPORT, Hiellel. Globalization, brain drain and development; ARSLAN, Casin et alii. A new profile of migrants in the aftermath of the recent economic crisis. 
En este contexto, México se distingue por figurar en los primeros planos de la migración mundial. En efecto, como se aprecia en el cuadro 5, en 1990 nuestro país se situó en el sexto sitio; mientras que en 2000 ascendió al segundo, casi a la par de la Federación Rusa que ocupó la primera posición; en 2010 pasó a la primera posición y en 2013, por razones a las que nos referiremos más adelante, descendió al segundo lugar, apenas por debajo de la India. Y si bien el país cuenta con una población inferior a la de la Federación Rusa y muy inferior a la de India, cabe preguntarnos ¿cuáles son las razones que han ubicado a México en la cima de la migración mundial?

\section{Cuadro 5 - Población emigrante según país de origen, países seleccionado, 1990, 2000, 2010 y 2013}

\begin{tabular}{|c|c|c|c|c|c|c|c|c|c|c|c|}
\hline \multicolumn{3}{|c|}{1990} & \multicolumn{3}{|c|}{2000} & \multicolumn{3}{|c|}{2010} & \multicolumn{3}{|c|}{2013} \\
\hline & Origen & $\begin{array}{l}\text { Población } \\
\text { emigrante }\end{array}$ & & Origen & $\begin{array}{l}\text { Población } \\
\text { emigrante }\end{array}$ & & Origen & $\begin{array}{l}\text { Población } \\
\text { emigrante }\end{array}$ & & Origen & $\begin{array}{l}\text { Población } \\
\text { emigrante }\end{array}$ \\
\hline & Mundo & & & Mundo & 174515733 & & Mundo & 214199193 & & Mundo & 231522215 \\
\hline $1^{\circ}$ & Fed. Rusa & 12749832 & $1^{\circ}$ & Fed. Rusa & 10702336 & $1^{\circ}$ & México & 12441703 & $1^{\circ}$ & India & 14166558 \\
\hline $2^{\circ}$ & Afganistán & 7295340 & $2^{\circ}$ & México & 10040849 & $2^{\circ}$ & India & 11398091 & $2^{\circ}$ & México & 13212220 \\
\hline $3^{\circ}$ & India & 6845565 & $3^{\circ}$ & India & 8120278 & $3^{\circ}$ & Fed. Rusa & 10725449 & $3^{\circ}$ & Fed. Rusa & 10832708 \\
\hline $4^{\circ}$ & Bangladesh & 5635489 & $4^{\circ}$ & Ucrania & 5714739 & $4^{\circ}$ & China & 8432427 & $4^{\circ}$ & China & 9342485 \\
\hline $5^{\circ}$ & Ucrania & 5575082 & $5^{\circ}$ & Bangladesh & 5695075 & $5^{\circ}$ & Bangladesh & 6476821 & $5^{\circ}$ & Bangladesh & 7757315 \\
\hline $6^{\circ}$ & México & 5043269 & $6^{\circ}$ & China & 5493899 & $6^{\circ}$ & Ucrania & 6356532 & $6^{\circ}$ & Paquistán & 5687563 \\
\hline
\end{tabular}

Fuente: UN-DESA.Tables of total migrant stock at mid-year by origin and by major area, region, country or area of destination. 2012 and 2013. Disponible en <http://www.un.org/en/development/desa/population/migration/ data/estimates2/estimatesage.shtml>. Consultado el 20.10.2015.

Lo primero que cabría destacar en relación con la interrogante planteada, es que la migración y sobre todo la migración hacia el país vecino del norte -a la sazón principal país receptor de migrantes del mundo- cuenta con una larga historia que data de finales del siglo XIX. No se trata, empero, de un fenómeno de crecimiento lineal. Por el contrario, como se aprecia claramente en la figura 3 (referida específicamente al flujo migratorio hacia los Estados Unidos), la escalada emigratoria del país comienza hacia finales de la década de 1970, periodo en el que la economía mundial entra en una etapa de recesión, la cual coincide con la desaceleración del proceso de sustitución de importaciones ante las dificultades para avanzar hacia la fase de sustitución de bienes intermedios y, sobre todo, de bienes de capital. Ello, a su vez, guarda relación con las vicisitudes por las que atraviesa la ciencia, la tecnología y la innovación en México, en tanto expresión del endeble curso seguido por el proceso de desarrollo del país. Pero la escalada migratoria se acentúa aún más y se torna exponencial cuando se abandona el modelo de sustitución de importaciones y se produce el viraje hacia el neoliberalismo con la implantación, a rajatabla, de los programas de ajuste estructural. Y más todavía: con la suscripción y 
puesta en marcha del Tratado de Libre Comercio de América del Norte (TLCAN), la escalada migratoria sube de tono hasta colocar a México en la cúspide de la migración mundial. Este curso, de suyo preocupante, se asocia a la desarticulación del aparato productivo nacional y la maquilización de la economía apuntalada por la reestructuración neoliberal, que instaura en el país lo que hemos caracterizado como el modelo exportador de fuerza de trabajo ${ }^{22}$, lo cual, lejos de reducir las brechas con los Estados Unidos, ha tendido a acentuarlas, como se aprecia claramente en el cuadro 6.

\section{Cuadro 6 - Asimetrías México-Estados Unidos en el contexto del TLCAN}

\begin{tabular}{lcccc}
\hline & \multicolumn{2}{c}{ México } & \multicolumn{2}{c}{ Estados Unidos } \\
\hline Asimetrías & $\mathbf{1 9 9 4}$ & $\mathbf{2 0 1 2}$ & $\mathbf{1 9 9 4}$ & $\mathbf{2 0 1 2}$ \\
\hline PIB per capta dólares corrientes & 4,54 & 9,24 & 26,82 & 48,45 \\
\hline Índices de la producción industrial & 71,74 & 101,65 & 80,49 & 121,13 \\
\hline $\begin{array}{l}\text { Gasto en investigación y } \\
\text { desarrollo (\% del PIB) }\end{array}$ & 0,29 & 0,48 & 2,48 & 2,90 \\
\hline $\begin{array}{l}\text { Salario manufactureros } \\
\text { (dólares por hora) }\end{array}$ & 2,10 & 2,60 & 12,00 & 9,30 \\
\hline Índice de Desarrollo Humano & 51 & 61 & 4 & 3 \\
\hline
\end{tabular}

Fuente: SIMDE. UAZ. Elaborado con base en INEGI, ENOE 4 trimestre 2012, Banco de información económica INEGI; U.S. Bureau of Labor Statistics BLS. Current Population Survey, 1994, 1995, 2011 y 2012.

\section{Figura 3 - Migración México-Estados Unidos}

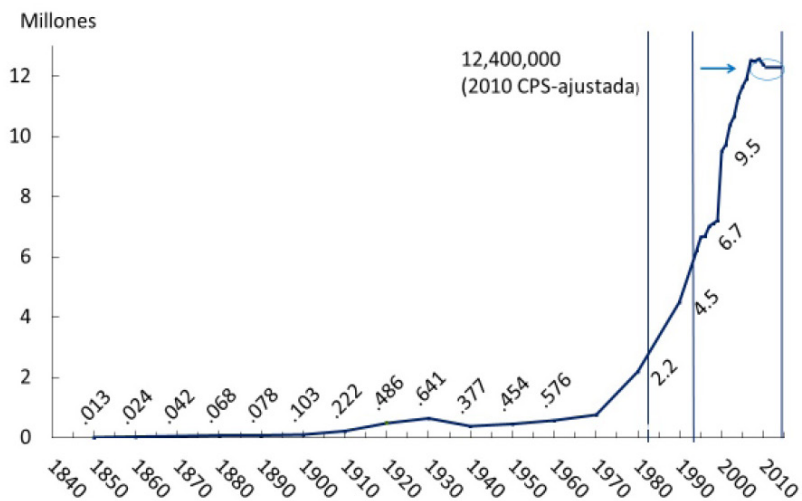

Fuente: Compilation from Decennial Censuses, 1850-1990; Pew Hispanic Center, 1994-2010 (PASSEL, Jeffrey S. y COHN, D’Vera. Unauthorized Immigrant Population: National and State Trends).

El panorama descrito quedaría incompleto si no se considera que a los 12 millones de mexicanos que radican en Estados Unidos habría que sumar

${ }^{22}$ CYPHER, James, DELGADO-WISE, Raúl. México a la deriva. Génesis, desempeño y crisis del Modelo Exportador de Fuerza de Trabajo. 
una comunidad de origen mexicano de poco más de 30 millones. Asimismo, no puede soslayarse, en tanto manifestación de las condiciones en las que se despliega la migración mexicana, que nuestro país cuenta también con el mayor número de migrantes indocumentados del planeta, los cuales se estiman en poco más de seis millones y que despunta también como el principal corredor de migración de tránsito del orbe, al tiempo que se ha convertido en un país de retorno migratorio forzado, debido fundamentalmente al incremento espectacular que han experimentado las deportaciones a lo largo de la administración del Presidente Barak Obama.

En este marco, uno de los rasgos más sobresalientes de la migración mexicana es el desbordante crecimiento que ha experimentado la migración altamente calificada desde 1990 y particularmente en lo que va del siglo XXI, como se aprecia en la figura 4.

Figura 4 - Crecimiento de la migración mexicana calificada 2000-2013
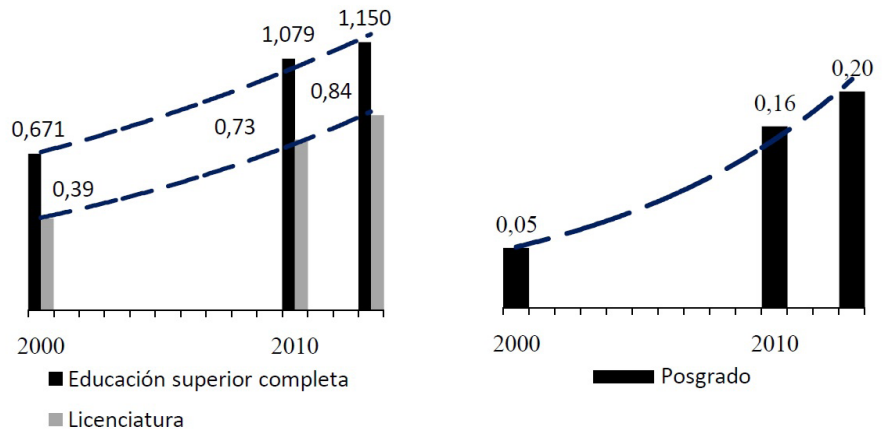

Fuente: SIMDE-UAZ. Estimación propia con base en Censos de países disponibles en IPUMS International del Minnesota Population Center, 2014 y UN-DESA, 2013; y U.S. Bureau of the Census, Percent Samples 1990, American Community Survey (ACS) 2000, 2010 y 2013.

Es pertinente agregar que México figura como el primer país de América Latina y sexto a nivel mundial, por su volumen de migrantes altamente calificados hacia países de la OECD ${ }^{23}$. El monto de profesionistas mexicanos con licenciatura, grado asociado a licenciatura, maestría y doctorado que residen en Estados Unidos asciende a 1.1 millones en 2011, lo que ubica al país en el segundo sitio de este tipo de inmigrantes en ese país (ver figura 5). A nivel de posgrado, el contingente de mexicanos -aunque relativamente bajo frente a China e India- más que se duplica en la última década, para alcanzar en 2011 un total de 129,027 con nivel de maestría y 12,026 con el grado de doctor. Este monto equivale a $17 \%$ de todos los posgraduados con los que cuenta el país ${ }^{24}$.

${ }^{23}$ DUMONT, Jean-Christophe, SPIELVOGEL, Gilles, WIDMAIER, Sarah. International Migrants in Developed, Emerging and Developing Countries. An Ex-tended Profile.

${ }^{24}$ TUIRÁN, Rodolfo, ÁVILA, José Luis. La migración calificada entre México-Estados Unidos. Desafíos y opciones de política. 
A ello, de acuerdo con los datos aportados por la American Community Survey, se agregan 2.5 millones profesionistas de origen mexicano nacidos en Estados Unidos, de los cuales 328,298 cuentan con maestría y 26,050 con doctorado. Si comparamos estos montos con la población altamente calificada que reside en México, la proporción de maestros y doctores de origen mexicano en Estados Unidos (inmigrantes y nacidos en ese país) resulta de enorme y sorprendente significación: $55.2 \%$ y $29.7 \%$ del total, respectivamente (ver figura 6 ).

\section{Figura 5 - Inmigrantes altamente calificados en Estados Unidos por país de origen, 2011}

Población extranjera de 25 años y más (millones)

1,3
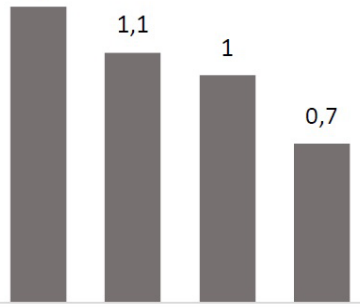

$\ln _{9}$
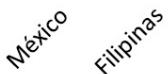<smiles>CC(C)C</smiles>
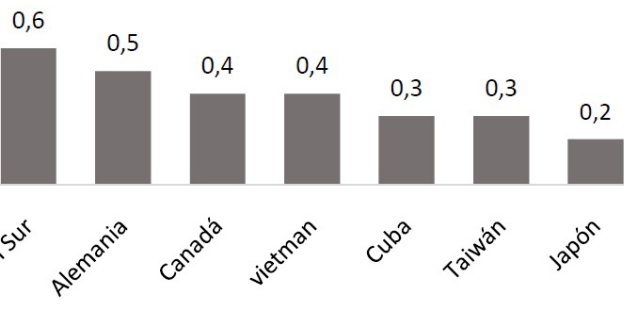

Fuente: SIMDE UAZ. Estimación con base en U.S. Bureau of Census, American Community Survey 2011.

\section{Figura 6 - Peso relativo de la población de origen mexicano con posgrado en Estados Unidos respecto de la radicada en México 2010-2011}

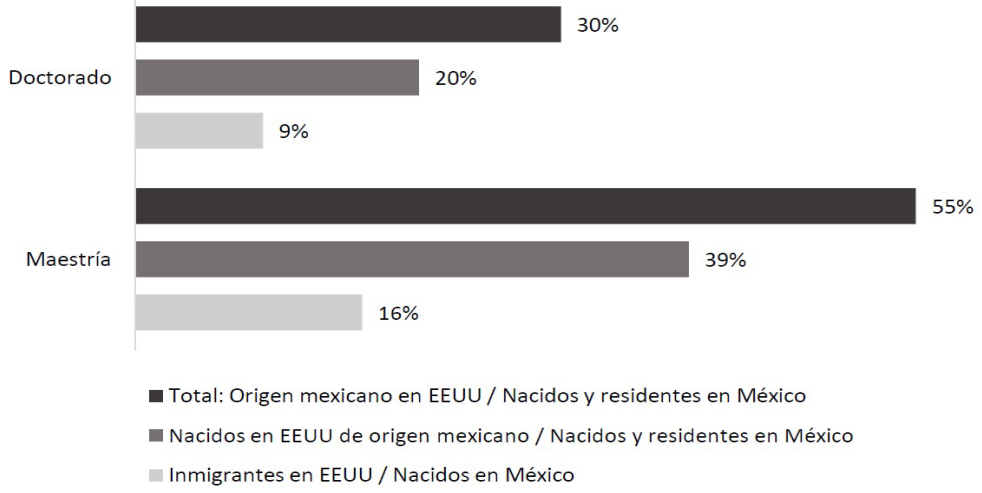

Fuente: SIMDE. UAZ. Estimación con base en INEGI, Censo de Población y Vivienda 2010 y U.S. Bureau of Census, American Community Survey, 2011. 
Más allá de estas cantidades, que de suyo son impactantes, es preciso destacar que apenas un $54.7 \%$ de nuestros connacionales se ocupan en actividades profesionales o gerenciales en los Estados Unidos (a nivel de posgrado la proporción se incrementa significativamente: $74.3 \%$ ) y que sus salarios, incluyendo los de los profesionistas de origen mexicano, tienden a ser más bajos que los del resto de los nativos e inmigrantes. A nivel de posgrado la situación salarial de los mexicanos y la población de origen mexicano en Estados Unidos sigue un patrón similar, aunque relativamente menos oneroso ${ }^{25}$.

A lo anterior se aúna la baja cobertura de educación superior que caracteriza al país (30\%), no obstante el ostensible incremento que ha tenido el posgrado en la última década, lo que pone de relieve no sólo una limitada formación de fuerza de trabajo altamente calificada, sino una creciente pérdida, desperdicio y desaprovechamiento de talentos para el desarrollo nacional. Esta situación adquiere particular relevancia al considerar la proporción de posgraduados mexicanos en computación y análisis de sistemas, ciencias e ingeniería de origen mexicano en Estados Unidos (inmigrantes y nacidos en ese país) en comparación con los que residen en México, la cual representa el 99\%, $66 \%$ y $47 \%$, respectivamente (véase figura 7 ) $^{26}$.

\section{Figura 7 - Peso relativo de la población de origen mexicano en con posgrado en Ciencias e Ingeniería en EU respecto de la radicada en México, 2010-2011}

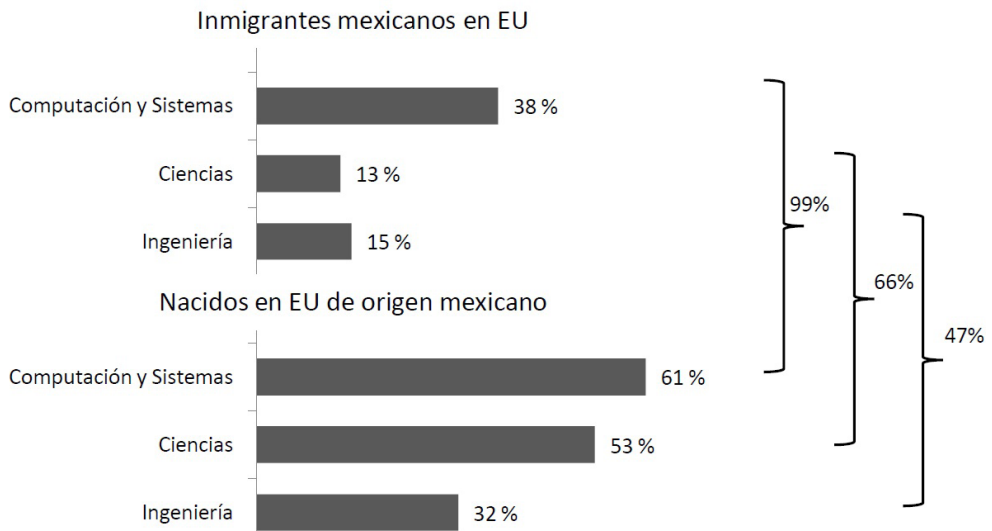

Fuente: SIMDE. UAZ. Estimación con base en INEGI, Censo de Población y Vivienda 2010 y U.S. Bureau of Census, American Community Survey, 2011.

${ }^{25}$ LOZANO-ASCENCIO, Fernando, GANDINI, Luciana, RAMÍREZ-GARCíA, Telésforo. Devaluación del trabajo de posgraduados en México y su relación con la migración internacional: el caso de los profesionistas en ciencia y tecnología.

${ }^{26}$ Para un análisis más detallado de este fenómeno, consúltese LOZANO-ASCENCIO, GANDINI, RAMÍREZ-GARCÍA, op. cit. 
Cabe agregar que este problema cobra dimensiones todavía mayores al considerar que de los posgraduados mexicanos, el segmento de mayor dinamismo -como se aprecia en el cuadro 7, referido a Estados Unidos- es el correspondiente a los que cuentan con el grado de Doctor.

\section{Cuadro 7 - Estados Unidos: tasa anual de crecimiento de la población nacida en México con posgrado}

\begin{tabular}{cccc}
\hline & Maestría & $\begin{array}{c}\text { Grado } \\
\text { Profesional }\end{array}$ & Doctorado \\
\hline $2000-2010$ & 7.6 & 0.6 & 13.4 \\
\hline $2010-2013$ & 4.9 & -1.1 & 6.1 \\
\hline $2000-2013$ & 7.0 & 0.2 & 11.7 \\
\hline
\end{tabular}

Fuente: SIMDE UAZ. Estimaciones propias con base en U.S. Bureau of Census. American Community Survey (ACS), 2000-2012 y Current Population Survey March supplement (CPS) $1994-1998$ y $2013-2014$.

Finalmente, es pertinente subrayar que a diferencia de la migración mexicana en general, que se concentra casi en su totalidad en Estados Unidos (98\%), la migración altamente calificada no obstante que mayoritariamente se ubica en ese país $(80 \%)$, acusa una relativa y creciente diversificación geográfica. En efecto, en una encuesta para el Consejo Nacional de Ciencia y Tecnología realizada a posgraduados mexicanos que radican en el exterior, en la que participaron los autores de este trabajo, se detectó la presencia de mexicanos en al menos 69 países de los cinco continentes.

\section{A manera de conclusión}

La reestructuración de los sistemas de innovación en el marco de la globalización neoliberal constituye un mirador privilegiado para analizar y comprender el significado e implicaciones de la migración altamente calificada tanto para los países de origen y destino, como para los principales sujetos involucrados: corporaciones multinacionales, universidades, centros de investigación y los propios migrantes. Desde este mirador se evidencia la inoperancia y superficialidad de las nociones de fuga de cerebros (brain drain), circulación de cerebros (brain circulation) y ganancia de cerebros (brain gain) como categorías analíticas supuestamente explicativas del fenómeno.

Retomando la noción de exportación de fuerza de trabajo empleada para caracterizar al modelo exportador que se instaura en México, queda claro que la dinámica de reestructuración de los sistemas de innovación antes referida se inscribe en este contexto. De hecho, se configura como una etapa avanzada o superior de la misma, que opera en una doble vertiente: la exportación directa vía migración de fuerza de trabajo altamente calificada y la exportación indirecta, mediante el establecimiento de maquiladoras científicas. Esta última 
vertiente es aun relativamente incipiente en el caso de México. Al respecto, Kevin Gallagher y Lyuba Zarsky ${ }^{27}$, en un estudio sólidamente fundamentado y bastante revelador, ponen de relieve que la inversión extranjera directa en tecnologías de la información realizada en el llamado Silicon Valley mexicano (ubicado en la zona metropolitana de Guadalajara), no generó un foco de innovación capaz de generar impactos multiplicadores sobre la economía mexicana y operó, más bien, bajo la modalidad de una economía de enclave.

En un sentido más profundo, es pertinente advertir que la exportación de fuerza de trabajo encierra una nueva modalidad de intercambio desigual en el horizonte norte-sur (y en nuestro caso, entre México y Estados Unidos), cuya comprensión resulta crucial para develar los procesos de transferencia de excedentes implicados en el entramado estratégico/estructural que se teje en torno a las cadenas globales de mercancías y que están en la base de la reinserción asimétrica y subordinada de la economía mexicana a la estadounidense. No obstante la centralidad que el concepto de intercambio desigual tuvo en décadas pasadas para explicar la dinámica del desarrollo desigual y desentrañar la naturaleza del vínculo entre países desarrollados y periféricos o emergentes -tanto en el pensamientos de la Comisión Económica para América Latina (CEPAL) como entre los teóricos de la dependencia-, su inclusión en los análisis del capitalismo contemporáneo permanece como una asignatura pendiente. Es importante tener presente que el grueso de la discusión sobre intercambio desigual estuvo y permanece circunscrita al análisis de la división internacional del trabajo que confiere a la periferia el papel de proveedora de materias primas y a los países desarrollados, el de suministradores de productos industrializados. Y si bien esta división continúa vigente para un número significativo de países periféricos, ha dejado de ser privativa de las relaciones norte-sur. Algunos países periféricos de reciente industrialización principalmente de la región asiática- fungen, cada vez más, como proveedores de bienes industrializados. Más importante aún resulta el hecho de que, a esta modalidad clásica de intercambio desigual, se ha agregado una nueva, que bajo la égida de la globalización neoliberal cobra progresiva centralidad: la exportación, directa e indirecta, de fuerza de trabajo.

Para incursionar en el análisis de esta modalidad, en su doble vertiente, es preciso advertir que involucra mecanismos de intercambio desigual mucho más desfavorables para la periferia que aquellos implicados en el intercambio de materias primas por bienes manufacturados. Por un lado, la exportación indirecta de fuerza de trabajo asociada a la participación de las naciones periféricas en calidad de apéndices de las cadenas globales de mercancías, conlleva una transferencia neta de las ganancias al exterior. Se trata de una

${ }^{27}$ GALLENGHER, Kevin, ZARSKY, Lyuba. The Enclave Economy: Foreign Investment and Sustainable Development in Mexico's Silicon Valley. 
forma extrema de intercambio desigual, ya que implica trasladar al exterior prácticamente la totalidad del excedente económico generado por la fuerza de trabajo empleada en las maquiladoras o plantas de ensamble. Mediante este mecanismo, que reedita la lógica del enclave exportador, se inhibe cualquier perspectiva de crecimiento económico y desarrollo derivada de las operaciones de exportación que, bajo el disfraz de exportaciones manufacture-ras, realiza la nación periférica. De hecho, su contribución efectiva al proceso de acumulación nacional se limita a una exigua derrama salarial -aprovechando los enormes diferenciales salariales entre países, en nuestro caso entre México y Estados Unidos- y, en el mejor de los casos, a un reducido impacto multiplicador por la vía del consumo. Más aún, la instalación y operación de plantas de ensamble en países periféricos, viene acompañada de subsidios y exenciones tributarias, con cargo al fondo de capital social del país en cuestión, así como de daños colaterales tales como el estrechamiento, diferenciación y precarización de los mercados laborales y la degradación ambiental. Otro aspecto de la exportación indirecta de fuerza de trabajo, que ha comenzado a cobrar fuerza en el contexto de los países periféricos o emergentes, es la creación de complejos científico/ tecnológicos vinculados, como vimos, a la reestructuración de los sistemas de innovación en algunos de los países de mayor desarrollo, con Estados Unidos a la cabeza. A través de estos complejos, que funcionan bajo esquemas de subcontratación, asociación u otras formas de partnership, se transfieren al exterior bienes intangibles que tienen un valor y un significado estratégico aún mayor que las ganancias netas provenientes de la maquila y las plantas de ensamble. Nos referimos a la transferencia de capacidad de desarrollo y progreso, lo cual se materializa en ventajas competitivas y capacidades para generar ganancias extraordinarias.

Por otra parte, la exportación directa de fuerza trabajo vía migración laboral implica la transferencia de los beneficios futuros anticipados que surgen de los gastos formativos y de reproducción social de la fuerza de trabajo que emigra, los cuales no son compensados por el flujo de remesas ${ }^{28}$. En términos demográficos, esta transferencia significa -para países periféricos que se ubican en un estadio avanzado de la transición demográfica- la exportación de su bono demográfico, es decir, de la población en edad productiva que sirve de soporte para el sostenimiento de la población infantil y los adultos mayores. En un sentido más profundo, esta transferencia implica la pérdida del más importante recurso para la acumulación de capital en el país de origen: su fuerza laboral. Más todavía, la exportación de fuerza de trabajo altamente calificada exacerba el problema, al reducir seriamente las capacidades del país de origen para

${ }^{28}$ DELGADO-WISE, Raúl, MÁRQUEZ, Humberto, RODRÍGUEZ, Héctor. Seis tesis para desmitificar el nexo entre migración y desarrollo. 
innovar en su propio beneficio e impulsar proyectos de desarrollo intensivos en tecnología.

Analizar estas nuevas modalidades de intercambio desigual plantea un desafío teórico, metodológico y empírico, ya que requiere de cambios en la percepción y caracterización de las categorías utilizadas convencionalmente para interpretar el capitalismo contemporáneo. Con todo, sin desconocer los significativos aportes de la CEPAL para avanzar en la comprensión de este fenómeno (sobre todo en lo concerniente al papel central otorgado al progreso técnico), es importante traer a colación que las teorías marxistas del intercambio desigual, en su doble vertiente: en sentido estricto y amplio, proveen un sólido y fértil piso conceptual para avanzar en la conceptualización de las modalidades emergentes de intercambio desigual implicadas en la exportación de fuerza de trabajo. Por un lado, el intercambio desigual en sentido estricto pone en el centro del análisis los diferenciales salariales derivados de los obstáculos a la movilidad poblacional (que, en términos más precisos, se refieren a los diferenciales en las tasas de plusvalor) y, por el otro, el intercambio desigual en sentido amplio agrega a estos diferenciales aquellos emanados de las diversas composiciones orgánicas del capital, i. e. los diferenciales en progreso científico/tecnológico, de los países involucrados. Tómese en consideración que la internacionalización del capital en el marco de la globalización neoliberal busca incesantemente abaratar los costos laborales -incluyendo aquellos relacionados con la fuerza de trabajo altamente calificada- y maximizar las transferencias de excedentes entre países periféricos y desarrollados, a partir, precisamente, de aprovechar y profundizar los diferenciales salariales.

Todo esto nos lleva a concluir que la exportación de fuerza de trabajo altamente calificada proveniente de países periféricos o emergentes, lejos de constituir una opción en la que todos ganan (win-win-win) -como lo sugiere la noción de circulación de talentos en boga- configura una nueva modalidad de dependencia, particularmente expoliadora y depredadora.

Ante este escenario, resulta urgente revertir las tendencias al desarrollo desigual prevalecientes y modificar radicalmente la lógica que orienta las dinámicas de innovación bajo la égida de la globalización neoliberal y, en general, del capital. Esto implica, entre otras cosas, avanzar hacia procesos de transformación social de gran calado que privilegien la satisfacción de las necesidades sociales; frenen la mercantilización y apropiación privada desenfrenada de la naturaleza, el trabajo y el dinero, y promuevan el derecho e institucionalización de los bienes comunes tangibles e intangibles, bajo los principios de emancipación del trabajo, supresión de los enclosures que emanan del derecho de propiedad, acceso irrestricto a servicios públicos y democracia social ${ }^{29}$.

${ }^{29}$ LAVAL, Christian, DARDOT, Pierre. Común. Ensayo sobre la revolución del siglo XXI. 


\section{Referencias}

ARSLAN, Casin; DUMONT, Jean-Christophe; KONE, Zovanga; MOULLAN, Yasser; OZDEN, Caglar; PARSONS, Christopher; XENOGIANI, Theodora. A new profile of migrants in the aftermath of the recent economic crisis. OECD Social, Employment and Migration Working Papers, n. 160, OECD Publishing, 2014. Disponible en <http://www.oecd.org/els/mig/WP160.pdf > . Consultado el 10.12.2015.

ARTUC, Erhan; DOCQUIER, Frédéric; ÖZDEN, Caglar; PARSONS, Christopher. A Global Assessment of Human Capital Mobility: The Role of Non-OECD Destinations. World Development, v. 65, 2015, p. 6-25.

BATTELLE. 2013 Global R\&D Funding Forecast. Columbus: Battelle, 2012. Disponible en <http://www.rdmag.com/sites/rdmag.com/files/GFF2013Final2013_reduced .pdf $>$. Consultado el 12.12.2015.

BATTELLE. 2014 Global R\&D funding forecast. Battelle-R\&D, 2013. Disponible en <http://www.battelle.org/docs/tpp/2014_global_rd_funding_forecast.pdf > . Consultado el 20.11.2015.

CHESBROUGH, Henry. Open Innovation: A New Paradigm for Understanding Industrial Innovation. In CHESBROUGH, Henry; VANHAVERBEKE, Wim; WEST, Joel (eds.). Open Innovation: Researching a New Paradigm, Oxford: Oxford University Press, 2008, p. 1-14.

CYPHER, James; DELGADO-WISE, Raúl. México a la deriva. Génesis, desempeño y crisis del Modelo Exportador de Fuerza de Trabajo. México: Editorial Miguel Ángel Porrúa, 2012.

DELGADO-WISE, Raúl; MARTIN, David. La economía política del arbitraje laboral global: capital y trabajo en el contexto de la globalización neoliberal. Problemas del Desarrollo, v. 46, n. 183, 2015, p. 13-32.

DELGADO-WISE, Raúl; MÁRQUEZ, Humberto; RODRÍGUEZ, Héctor. Seis tesis para desmitificar el nexo entre migración y desarrollo. Migración y Desarrollo, v 6, n. 12, 2009, p. 27-52.

DOCQUIER, Frédéroc; RAPOPORT, Hiellel. Globalization, brain drain and development. IZA Discussion Paper, n. 5590, Marzo 2011. Disponible en <http:// ftp.iza.org/dp5590.pdf>. Consultado el 11.11.2015.

DUMONT, Jean-Christophe; SPIELVOGEL, Gilles; WIDMAIER, Sarah. International Migrants in Developed, Emerging and Developing Countries. An Ex-tended Profile. Paris: OECD, 2010.

FOLADORI, Guillermo. Ciencia ficticia. Estudios Críticos del Desarrollo, ano IV, n. 7, 2014, p. 41-66.

FREEMAN, Richard. The new global labor market. Focus, v. 26, n. 1, 2008, p. 1-6. GALAMA, Titus; HOSEK, James. U.S. Competitiveness in Science and Technology. Santa Mónica: RAND Corporation, 2008. Disponible en <http://www.rand.org/content/ dam/rand/pubs/monographs/2008/RAND_MG674.pdf>. Consultado el 10.12.2015. 
GALLENGHER, Kevin; ZARSKY, Lyuba. The Enclave Economy: Foreign Investment and Sustainable Development in Mexico's Silicon Valley. Cambridge, Massachusetts: MIT Press, 2007.

ILO (INTERNATIONAL LABOR ORGANIZATION). Mainstreaming of Migration in Development Policy and Integrating Migration in the Post-2015 UN Development Agenda. 2015. Disponible en <http://www.ilo.org/wcmsp5/groups/public/--ed_protect/---protrav/---migrant/documents/genericdocument/wcms_220084. pdf $>$. Consultado el 11.12.2015.

IOM (INTERNATIONAL ORGANIZATION FOR MIGRATION). Global Migration Trends: an overview. Geneva: International Organization for Migration, 2014. Disponible en <http://gmdac.iom.int/sites/default/files/Global_Migration_ Trends_2014_PDF.pdf >. Consultado el 12.10.2015.

LAVAL, Christian; DARDOT, Pierre. Común. Ensayo sobre la revolución del siglo XXI. Barcelona: Gedisa editorial, 2014.

LOZANO-ASCENCIO, Fernando; GANDINI, Luciana. Migración calificada y desarrollo humano en América Latina y el Caribe. Revista Mexicana de Sociología, v. 73, n. 4, 2011, p. 675-713.

LOZANO-ASCENCIO, Fernando; GANDINI, Luciana; RAMÍREZ-GARCÍA, Telésforo. Devaluación del trabajo de posgraduados en México y su relación con la migración internacional: el caso de los profesionistas en ciencia y tecnología. Migración y Desarrollo, v. 13, n. 25, 2015, p. 61-89.

MIGUÉLEZ, Ernest; FINK, Carsten. Measuring the international mobility of inventors: a new database. WIPO Economic Research Working Paper No. 8, 2013.

MORIS, Franciso; KANNANKUTTY, Nirmala. New employment statistics from 2008 Business R\&D and Innovation Survey. National Science Foundation, 2008. Disponible en < http://www.nsf.gov/statistics/infbrief/nsf10326/> . Consultado el 20.10.2015.

NATIONAL SCIENCE BOARD. Science and Engineering Indicators Digest. Arlington: The National Science Board, 2012.

OECD (ORGANISATION FOR ECONOMIC CO-OPERATION AND DEVELOPMENT). Open Innovation in Global Networks. Copenhaguen: OECD, 2008.

PASSEL, Jeffrey S.; COHN, D'Vera. Unauthorized Immigrant Population: National and State Trends, 2010. Washington, D.C.: Pew Hispanic Center, 2011. Disponible en <http://www.pewhispanic.org/files/reports/133.pdf > . Consultado el 20.10.2015.

RAMIS, Álvaro. Los bienes comunes intangibles en el capitalismo cognitivo. Recerca. Revista de Pensament i Anàlisi, n. 15, 2014, p. 109-129.

RAND CORPORATION. U.S. Competitiveness in Science and Technology. Santa Monica, CA: Rand Corporation, 2008.

SAXENIAN, Annelee. Regional Advantage: Culture and Competition in Silicon Valley and Route 128. Boston: Harvard University Press, 1996. 
SAXENIAN, Annelee. Local and Global Networks of Immigrant Professsionals in Silicon Valley. San Francisco: Public Policy Institute of California, 2002.

SMITH, Gina. Can the U.S. patent system be saved? ComputerWold, 2012. Disponible en <http://www.computerworld.com/article/2505817/it-management/can-theu-s--patent-system-be-saved-.html>. Consultado el 10.10.2015.

STURGEON, Timothy J. What really goes on in Silicon Valley? Spatial clustering and dispersal in modular production networks. Journal of Economic Geography, v. 3, n. 2, 2003, p. 199-225.

TUIRÁN, Rodolfo; ÁVILA, José Luis. La migración calificada entre México-Estados Unidos. Desafíos y opciones de política. Migración y Desarrollo, v. 11, n. 3, 2013, p. 43-63.

USPTO (UNITED STATES PATENT AND TRADEMARK OFFICE). Patenting by Organizations. 2012. Disponible en <http://www.uspto.gov/web/offices/ac/ido/ oeip/taf/topo_12.pdf >. Consultado el 12.12.2015.

VERCELLY, Ariel; THOMAS, Hernán. Repensando los bienes comunes: análisis soco-técnico sobre la construcción y regulación de los bienes comunes. Scientiae Studia, v. 6, n. 3, 2008, p. 427-442.

Recibido para publicación en 01.03.2016

Aceptado para publicación en 27.07.2016

Received for publication in March 01st, 2016

Accepted for publication in July $27^{\text {th }}, 2016$

ISSN impresso 1980-8585

ISSN eletrônico 2237-9843

http://dx.doi.org/10.1590/1980-85852503880004710 